\title{
Placenta as a newly identified source of hematopoietic stem cells
}

\author{
Lydia K. Lee ${ }^{1,2}$, Masaya Ueno², Ben Van Handel², and Hanna K. A. Mikkola 2,3 \\ ${ }^{1}$ Department of Obstetrics and Gynecology, University of California, Los Angeles, Los Angeles, CA, \\ USA \\ 2Department of Molecular, Cell and Developmental Biology, University of California, Los Angeles, \\ Los Angeles, CA, USA \\ ${ }^{3}$ Eli and Edythe Broad Center of Regenerative Medicine and Stem Cell Research, University of \\ California, Los Angeles, Los Angeles, CA, USA
}

\section{Abstract}

Purpose of review-The lifelong stream of all blood cells originates from the pool of hematopoietic stem cells (HSC) generated during embryogenesis. Given that the placenta has been recently unveiled as a major hematopoietic organ that supports HSC development, the purpose of this review is to present current advances in defining the origin and regulation of placental HSCs.

\begin{abstract}
Recent findings-The mouse placenta has been shown to have the potential to generate multipotential myelo-lymphoid hematopoietic stem/progenitor cells (HS/PC) de novo. The cellular origin of HSCs generated in the placenta and other sites has been tracked to the hemogenic endothelium by using novel genetic and imaging-based cell-tracing approaches. Transplantable, myelo-lymphoid HS/PCs have also been recovered from the human placenta throughout gestation.
\end{abstract}

Summary-The discovery of the placenta as a major organ that generates HSCs and maintains them in an undifferentiated state provides a valuable model to further elucidate regulatory mechanisms governing HSC emergence and expansion during mouse and human development. Concurrent efforts to optimize protocols for placental banking and HSC harvesting may increase the therapeutic utility of the human placenta as a source of transplantable HSCs.

\section{Keywords}

placenta; hematopoietic stem cell; hemogenic endothelium; blood flow; lineage tracing

\section{Introduction}

Lifelong production of all blood cell types is sustained by the multipotent hematopoietic stem cells (HSC). HSCs are formed only during a narrow time window in embryonic development, after which their pool is maintained by self-renewing cell divisions. However, HSCs are not the first hematopoietic cells to appear during development, as early embryonic survival depends on the immediate production of mature blood cells. The challenge of rapidly generating the differentiated blood cells while concomitantly protecting developing HSCs from premature differentiation is met by segregating embryonic hematopoiesis into multiples waves that occur in different anatomical compartments.

\footnotetext{
Correspondence to Hanna K. A. Mikkola, Department of Molecular, Cell and Developmental Biology, Eli and Edythe Broad Center of Regenerative Medicine and Stem Cell Research, University of California, Los Angeles, 615 Charles E Young Drive South, BSRB, Room 451B, Los Angeles, CA, 90095, Tel: 310-825-2565, Fax: 310-206-5553, hmikkola@ mcdb.ucla.edu.
} 
The first, transient populations of blood progenitors giving rise to the primitive erythroblasts and definitive myelo-erythroid cells --required to ensure fetal oxygen delivery, tissue remodeling and defense--, are produced in the blood islands of the extra-embryonic yolk sac $(1,2)$. As the conceptus develops, other vascular tissues are recruited for HSC production. Once generated,HSCs are sequestered into the fetal liver for their expansion and maturation before establishing lifelong residence in the bone marrow (3). The aorta-gonad-mesonephros (AGM) region and the umbilical and vitelline arteries have been regarded as the sites of HSC emergence $(4,5)$. However, as the fetal liver cannot generate HSCs in situ and the number of HSCs found in the liver far exceeds the quantity formed in the AGM, the question was raised whether the yolk sac could also contribute to the pool of fetal liver HSCs, or whether another yet unidentified organ could participate in this process. Recent studies have revealed that the placenta is a major hematopoietic organ contributing to both generation and expansion of multipotential hematopoietic stem/progenitor cells (HS/PC). Here, we review how hematopoietic activity in the placenta was discovered in mice and humans, and discuss the recent findings on the cellular origin and regulation of placental HSCs.

\section{Discovery of hematopoietic stem cells in the mouse placenta}

Evidence of hematopoietic activity in the placenta was introduced by early reports documenting that the mouse placenta contains clonogenic hematopoietic precursors capable of rescuing anemia or triggering graft-versus-host disease after transplantation (6-8). Although the placenta was overlooked as a potential hematopoietic organ for decades, newfound interest in its role in blood formation has awakened in light of recent findings confirming that the placenta possesses intrinsic hematopoietic properties.

The hypothesis that the placenta could bear de novo hematopoietic activity stemmed from chick-to-quail grafting experiments, which revealed the presence of multipotent hematopoietic cells in the avian allantois (9). The allantois is a mesodermal appendage that functions in oxygen and nutrient exchange in avian embryos, analogous to the mammalian placenta. As the mammalian allantois gives rise to the umbilical cord and placental vasculature, it was hypothesized that these tissues could be engaged in hematopoiesis. A screen for hematopoietic activity across extra- and intraembryonic sites in mid-gestation embryos revealed multipotent progenitors in the placenta at the 20 somite-pair stage (approximately E9.0); that is, after similar progenitors were detectable in the yolk sac and the caudal half of the embryo but before the fetal liver (10). Subsequent studies confirmed that the placenta harbors bona fide HSCs that are able to generate all blood cell types upon serial transplantation into lethally irradiated adult mice $(11,12)$. Transplantation assays detected the first HSCs in the placenta at E10.5-11.0, concurrently with the AGM. Placental HSC activity increased rapidly by E12.5-13.5. At this time, the placenta harbored 15-fold more HSCs than the AGM or the yolk sac, whose repopulating units remained low. The number of HSCs in the liver increased concomitantly with the placenta (11), rising through late gestation even while the placental HSC pool declined. As the placenta is directly upstream of the liver in fetal circulation, these findings pointed to the placenta as a major contributor of HSCs seeding the liver.

Transplantation of FACS purified cells from the placenta confirmed that its HSCs at E12.5 displayed the classical surface phenotype of actively cycling fetal HSCs, expressing CD34 and c-Kit (11). Interestingly, another study presented E12.0 placental cells that were able to engraft

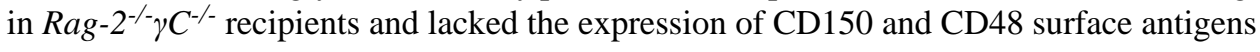
(13). This finding implies that although these $\mathrm{CD} 150^{-} \mathrm{CD} 48^{-} \mathrm{HSCs}$ are capable of multilineage engraftment, they may be phenotypically more immature than the $\mathrm{CD} 150^{+} \mathrm{CD} 48^{-} \mathrm{HSCs}$ found in the fetal liver later in development. Phenotypic maturation also occurs with respect to VEcadherin, which is expressed on endothelium and nascent HSCs (14), but this cell-surface protein is rapidly downregulated upon HSC colonization of the fetal liver and is absent from 
bone marrow HSCs $(15,16)$. These results suggest that in addition to changes in anatomic localization of HSCs, the dynamic process of HSC development also involves transitions in cell surface phenotype.

\section{Hematopoietic activity in the human placenta}

Because hematopoiesis is highly conserved in vertebrates, the discovery of HSCs in the mouse placenta attracted interest in the hematopoietic potential of the human placenta. Recent studies have provided evidence that the human placenta harbors hematopoietic activity throughout gestation $\left(17^{*}-19\right)$. It is important to note that two different systems are used to denote the age of a human conceptus: the developmental age $\left(18^{*}\right)$ calculated as the number of weeks from conception, and the clinical gestational age $\left(17^{*}, 19\right)$, which is 2 weeks more than the developmental age. One group reported that $\mathrm{CD} 34^{++} \mathrm{CD} 45^{\text {low }}$ placental cells could generate myeloid cells with some erythroid derivatives in methylcellulose assays, as well as natural killer and B cells in liquid cultures. Although the total number of $\mathrm{CD} 34^{++} \mathrm{CD} 45^{\text {low }}$ cells increases with placental mass asgestational age advances, the frequency of these cells peaks at 5-8 weeks and declines sharply at 9 weeks of gestation (i.e., 3-6 weeks and 7 weeks of developmental age, respectively) $\left(17^{*}, 19\right)$. This was reminiscent of the kinetics of HSCs in the mouse placenta (11). Residence of long-term reconstituting HSCs in human placentas was demonstrated in another study by performing transplantation assays of sorted $\mathrm{CD} 34^{+}$cells into irradiated NOD-SCID or $\operatorname{Rag} \gamma \mathrm{C}^{-{ }_{-}^{-}}$mice $\left(18^{*}\right)$. Multilineage reconstitution was observed from 9 weeks after conception until full-term delivery, while engraftment of human cells could be detected by PCR as early as 6 developmental weeks ( 11 and 8 weeks of gestational age, respectively). Although more work needs to be done to define the origin of HSCs in the human placenta, these studies have uncovered the human placenta as a robust and more accessible hematopoietic organ that can be used to investigate HSC ontogeny and regulation during human development.

Furthermore, placental HSCs may provide an additional source of HSCs for transplantation. Since the pioneering clinical studies that established the umbilical cord blood as a source of transplantable HSCs two decades ago, there have been continuous efforts to enhance the methods for the collection, cryopreservation and recovery of viable cells $(20,21)$. It is possible that in combination with the HSCs harvested from the cord blood, HSCs obtained from the placenta proper may provide sufficient HSCs to transplant adult patients.

\section{Origin of hematopoietic stem cells in the placenta}

Although functional assays suggested that the placenta provides a supportive niche for HSCs, these assays alone could not determine definitively whether placental HSCs emerge in situ or migrate from other hematopoietic sites. Since fetal blood circulation is directed from the dorsal aorta through the placenta to the fetal liver, most HSCs generated in the AGM traverse the placenta along their journey towards the liver and may pause in placental niches for expansion, there by contributing to the total placental HSC count.

In order to evaluate the capacity of the placenta to generate HSCs de novo, it is important to understand its ontogeny and structure $(22,23)$. The placenta is composed of two main tissues, the trophoblast and the mesoderm. The trophoblast lineage (trophectoderm) segregates from the embryonic lineage (inner cell mass) before implantation at the blastocyst stage (E3.5 in mice). Trophoblasts comprise the most abundant cell type in the placenta and line the maternal blood spaces, whereas the mesoderm-derived allantois generates the feto-placental vasculature. The allantois grows from the posterior primitive streak into the exocoelomic cavity and fuses with the chorion, inducing the extensive branching of the fetal vasculature into the trophoblast layer. This chorioallantoic patterning results in the formation of the labyrinthine region in the 
murine placenta and the chorionic villi in the human placenta, which are homologous sites of fetal-maternal exchange (See Figure 1).

To investigate whether the placental mesodermal tissues possess endogenous hematopoietic potential, mouse allantois and chorion were isolated prior to the establishment of circulation and subjected to explant or stroma co-culture assays. Two independent studies reported the capacity of these mesodermal tissues to generate myeloid and definitive erythroid cells in culture, providing evidence that placental tissues devoid of any circulating cells indeed had intrinsic hematopoietic potential $(24,25)$. Further evidence that the placenta is an autonomous site of origin of multipotential HS/PCs was provided by studies using $\mathrm{Ncxl}^{-/-}$mouse embryos, which lack blood flow due to the lack of heartbeat (26). Even in the absence of circulating contributors, cells expressing CD41 (a marker of nascent hematopoietic cells) were found to emerge from the lumen of large fetal blood vessels in the placenta at E10.0. Most importantly, placental tissues possessed the ability to generate both myelo-erythroid as well as B and T lymphoid cells, documenting the broad differentiation potential that is characteristic of HSCs. The results were comparable to the observed multilineage hematopoietic potential in the caudal half of the embryo proper (which contains the developing AGM) as well as in the yolk sac. Since the $\mathrm{NCx}^{-/}$embryos become developmentally retarded and die before E10.5, it was not possible to assess the engraftment and self-renewal potential of these cells by transplantation into adult recipients. Nevertheless, these studies provide strong evidence that the placenta parallels the AGM and the yolk sac in its intrinsic capacity to generate multipotential HS/PCs de novo.

Not only the anatomical source but also the cellular origin of HSCs has been a controversial topic. It has been postulated that the HSCs derive from a bipotent hemato-endothelial precursor (the hemangioblast) or a specialized endothelial cell (the hemogenic endothelium). One opposing theory has suggested that HSCs are specified within the tissue encapsulating the vascular endothelium and migrate through the vessel wall to be released into the blood stream (27). Recent studies using imaging techniques and conditionally targeted mouse models for lineage tracing suggest a unifying pathway whereby the hemangioblast first gives rise to hemogenic endothelium, which in turn generates HS/PCs. One study used time-lapse imaging of ES cell in vitro differentiation to document the development of hematopoietic progenitors from blast colonies, the in vitro equivalents of the hemangioblasts $\left(28^{*}\right)$. The cellular morphology and immunophenotype suggested that hemangioblasts first undergo $\mathrm{Scl}$ dependent establishment of hemogenic endothelial intermediates before giving rise to committed blood cells. Another study confirmed the transition of individual ES cell-derived hemangioblasts to hematopoietic cells via an endothelial intermediate using an imaging-based fate tracing approach that allowed monitoring of single cells and their progeny $\left(29^{*}\right)$. The role of the hemogenic endothelium as a source of HSCs was verified in vivo using a conditional deletion of Runxl (a transcriptional factor essential for HSC formation) from cells expressing the vascular endothelial cadherin (VEC) gene(30**). At E9.5, VEC is expressed in the vasculature throughout the conceptus, including the developing placental labyrinth. While excision of Runxl from VEC+ cells impaired HS/PC development, its excision from Vav+ hematopoietic cells no longer caused this defect. This indicated that Runxl is essential for HS/ PC development during a short developmental window (the time between the onset of VEC and $\mathrm{Vav}$ expression), providing evidence of the hemogenic endothelium as the HSC precursor. Likewise, when the vasculature and the perivascular mesenchyme were labeled with the inducible VEC Cre line and the myocardin Cre line, respectively, it was shown that HSCs in the AGM, placenta and yolk sac were traced to the VEC+ endothelial fraction $\left(31^{* *}\right)$. Although these studies strongly suggest that a crucial commitment step to HSC fate occurs during the hemogenic endothelial stage, they do not exclude the possibility that the hemogenic endothelium could develop from a mesenchymal precursor that does not express myocardin. 


\section{Hematopoietic microenvironment in the placenta}

In addition to the progress made in understanding the cellular origin of HSCs, current reports provide novel insights into signals regulating HSC development in the microenvironment. Recent studies demonstrate that blood flow and shear stress are critical for efficient HSC formation in the placenta. $\mathrm{Ncxl}^{-/-}$embryos, which lack a heartbeat, show reduced numbers of nascent CD41+ hematopoietic cells in the placental vasculature (26). Although the lack of circulating HS/PCs could result in decreased hematopoietic progenitors, prominent clusters of round cells expressing the endothelial marker CD31, and occasionally the hematopoietic marker CD41, were observed in the lumens of the large vessels in the $\mathrm{Ncxl}^{-/-}$placenta. It was hypothesized that these endothelial cell clusters represent hemogenic intermediates, which are unable to emerge in the absence of blood flow and thus accumulate in the placental vessels. The relationship between blood flow and shear stress was showed mechanistically in the zebrafish system. In Sih (silent heart) mutant zebrafish embryos, which also lack a heartbeat, the absence of blood flow impaired arterial identity and HSC formation in the AGM due to defective nitric oxide signaling (32**). A similar phenotype was found in wild-type embryos where blood vessel tone was altered with small molecules. The induction of shear forces has also been shown to enhance Runxl expression and hematopoietic potential in cells derived from mouse ES cells and embryos (33*). These results implicate hemodynamic forces as a conserved modulator of HSC development.

Although the molecular cues that regulate hematopoiesis in the placenta remain largely undefined, many cell types likely contribute to this supportive microenvironment. In mice, Runx $1^{+}$HS/PCs undergoing mitosis localized to small vessels in the labyrinthine region, suggesting that local signals support HS/PC proliferation (26) (See Figure 1). In human tissues, $\mathrm{CD} 34^{+} \mathrm{HS} / \mathrm{PCs}$ were found in the stroma and vasculature of the chorionic villi, the region analogous to the murine labyrinth $\left(17^{*}, 18^{*}, 26\right)$. The ability of trophoblasts to secrete mitogens and angiogenic factors, in addition to their proximity to placental blood vessels, makes them intriguing candidates for niche cells (34). Trophoblast-derived signals may affect HSCs by direct contact, or indirectly by establishing a vascular/perivascular niche where HSCs reside. Perivascular cells and/or mesenchymal stem cells (MSCs) in other hematopoietic organs have been associated with HSC supportive activity (35). Cells with pericyte characteristics capable of expanding CFU-GM and CFU-Mix progenitors during co-culture were identified as putative niche cells in the human placenta $\left(18^{*}\right)$. One study detected cells containing MSC properties with perivascular cells in the villi of second and third trimester human placentas (36). Beyond identifying the cellular composition of placental microenvironment, it is also important to understand the physiological changes that occur in the placenta during development. For example, changes in local oxygen levels are also likely to impact HSC fate during embryogenesis. It is known that hypoxia during early gestation promotes not only vasculogenesis and establishment of arterial endothelium (37), but also trophoblast proliferation (38). These data highlight the dynamic and multifaceted composition of the placental hematopoietic microenvironment, and provide new avenues to explore the complex regulation of HSC emergence and expansion.

\section{Conclusion}

The limited supply of HSCs for therapeutic use remains as one of the most pressing challenges in treating patients with hematopoietic malignancies and inherited blood disorders. The placenta provides another source of HSCs that can be harvested to supplement the circulating HSCs obtained from umbilical cord blood. The term placenta is an attractive source for HSCs because its procurement is non-invasive and it is routinely discarded. Furthermore, the first trimester placenta provides an accessible model for studying the earliest stages of HSC development. Improved understanding of the molecular and cellular mechanisms that govern 
HSC development in the placenta at different stages may ultimately provide effective tools to enhance protocols for HSC harvesting, ex vivo expansion, and generation from pluripotent cells.

\section{Acknowledgments}

This work was supported in part by grants from the National Institute of Health (NIH HL097766-01) and California Institute for Regenerative Medicine (CIRM RN1-00557-1 and RS1-00420-1) for H.K.A.M, California Institute for Regenerative Medicine Training Grant (CIRM TG2-01169) for L.K.L, the Ruth Kirschstein National Research Service Award (GM007185) for B.V.H and JSPS Postdoctoral Fellowship for M.U.

\section{References}

1. Lux CT, Yoshimoto M, McGrath K, Conway SJ, Palis J, Yoder MC. All primitive and definitive hematopoietic progenitor cells emerging before E10 in the mouse embryo are products of the yolk sac. Blood 2008 Apr 1;111(7):3435-8. [PubMed: 17932251]

2. Palis J. Ontogeny of erythropoiesis. Curr Opin Hematol 2008 May;15(3):155-61. [PubMed: 18391778]

3. Mikkola HK, Orkin SH. The journey of developing hematopoietic stem cells. Development 2006 Oct; 133(19):3733-44. [PubMed: 16968814]

4. de Bruijn MF, Speck NA, Peeters MC, Dzierzak E. Definitive hematopoietic stem cells first develop within the major arterial regions of the mouse embryo. EMBO J 2000 Jun 1;19(11):2465-74. [PubMed: 10835345]

5. Medvinsky A, Dzierzak E. Definitive hematopoiesis is autonomously initiated by the AGM region. Cell 1996 Sep 20;86(6):897-906. [PubMed: 8808625]

6. Dancis J, Samuels BD, Douglas GW. Immunological competence of placenta. Science 1962 May 4;136:382-3. [PubMed: 13883350]

7. Dancis J, Jansen V, Brown GF, Gorstein F, Balis ME. Treatment of hypoplastic anemia in mice with placental transplants. Blood 1977 Oct;50(4):663-70. [PubMed: 901940]

8. Melchers F. Murine embryonic B lymphocyte development in the placenta. Nature 1979 Jan 18;277 (5693):219-21. [PubMed: 317835]

9. Caprioli A, Jaffredo T, Gautier R, Dubourg C, Dieterlen-Lievre F. Blood-borne seeding by hematopoietic and endothelial precursors from the allantois. Proc Natl Acad Sci U S A 1998 Feb 17;95 (4):1641-6. [PubMed: 9465069]

10. Alvarez-Silva M, Belo-Diabangouaya P, Salaun J, Dieterlen-Lievre F. Mouse placenta is a major hematopoietic organ. Development 2003 Nov;130(22):5437-44. [PubMed: 14507780]

11. Gekas C, Dieterlen-Lievre F, Orkin SH, Mikkola HK. The placenta is a niche for hematopoietic stem cells. Dev Cell 2005 Mar;8(3):365-75. [PubMed: 15737932]

12. Ottersbach K, Dzierzak E. The murine placenta contains hematopoietic stem cells within the vascular labyrinth region. Dev Cell 2005 Mar;8(3):377-87. [PubMed: 15737933]

13. McKinney-Freeman SL, Naveiras O, Yates F, Loewer S, Philitas M, Curran M, et al. Surface antigen phenotypes of hematopoietic stem cells from embryos and murine embryonic stem cells. Blood 2009 Jul 9;114(2):268-78. [PubMed: 19420357]

14. Kim I, Yilmaz OH, Morrison SJ. CD144 (VE-cadherin)is transiently expressed by fetal liver hematopoietic stem cells. Blood 2005 Aug 1;106(3):903-5. [PubMed: 15831702]

15. Taoudi S, Gonneau C, Moore K, Sheridan JM, Blackburn CC, Taylor E, et al. Extensive hematopoietic stem cell generation in the AGM region via maturation of VE-cadherin+CD45+ pre-definitive HSCs. Cell Stem Cell 2008 Jul 3;3(1):99-108. [PubMed: 18593562]

16. Taoudi S, Morrison AM, Inoue H, Gribi R, Ure J, Medvinsky A. Progressive divergence of definitive haematopoietic stem cells from the endothelial compartment does not depend on contact with the foetal liver. Development 2005 Sep;132(18):4179-91. [PubMed: 16107475]

17*. Barcena A, Kapidzic M, Muench MO, Gormley M, Scott MA, Weier JF, et al. The human placenta is a hematopoietic organ during the embryonic and fetal periods of development. Dev Biol 2009 Mar 1;327(1):24-33. [PubMed: 19073167] This study reports that the human placenta contains 
multipotent hematopoietic progenitors with myelo-erythroid and lymphoid potential and localizes placental HS/PCs in the chorionic villi.

18*. Robin C, Bollerot K, Mendes S, Haak E, Crisan M, Cerisoli F, et al. Human placenta is a potent hematopoietic niche containing hematopoietic stem and progenitor cells throughout development. Cell Stem Cell 2009 Oct 2;5(4):385-95. [PubMed: 19796619] Human placental CD34+ cells were transplanted into immunocompromised mice and evidence of multilineage engraftment of human cells was demonstrated from 9 weeks of development onwards.

19. Barcena A, Muench MO, Kapidzic M, Fisher SJ. A new role for the human placenta as a hematopoietic site throughout gestation. Reprod Sci 2009 Feb;16(2):178-87. [PubMed: 19208786]

20. Broxmeyer HE, Cooper S, Hass DM, Hathaway JK, Stehman FB, Hangoc G. Experimental basis of cord blood transplantation. Bone Marrow Transplant 2009 Nov;44(10):627-33. [PubMed: 19802026]

21. Serikov V, Hounshell C, Larkin S, Green W, Ikeda H, Walters MC, et al. Human term placenta as a source of hematopoietic cells. Exp Biol Med (Maywood) 2009 Jul;234(7):813-23. [PubMed: 19429852]

22. Rossant J, Cross JC. Placental development: lessons from mouse mutants. Nat Rev Genet 2001 Jul; 2(7):538-48. [PubMed: 11433360]

23. Georgiades P, Ferguson-Smith AC, Burton GJ. Comparative developmental anatomy of the murine and human definitive placentae. Placenta 2002 Jan;23(1):3-19. [PubMed: 11869088]

24. Zeigler BM, Sugiyama D, Chen M, Guo Y, Downs KM, Speck NA. The allantois and chorion, when isolated before circulation or chorio-allantoic fusion, have hematopoietic potential. Development 2006 Nov;133(21):4183-92. [PubMed: 17038514]

25. Corbel C, Salaun J, Belo-Diabangouaya P, Dieterlen-Lievre F. Hematopoietic potential of the prefusion allantois. Dev Biol 2007 Jan 15;301(2):478-88. [PubMed: 17010964]

26. Rhodes KE, Gekas C, Wang Y, Lux CT, Francis CS, Chan DN, et al. The emergence of hematopoietic stem cells is initiated in the placental vasculature in the absence of circulation. Cell Stem Cell 2008 Mar 6;2(3):252-63. [PubMed: 18371450]

27. Bertrand JY, Giroux S, Golub R, Klaine M, Jalil A, Boucontet L, et al. Characterization of purified intraembryonic hematopoietic stem cells as a tool to define their site of origin. Proc Natl Acad Sci U S A 2005 Jan 4;102(1):134-9. [PubMed: 15623562]

28*. Lancrin C, Sroczynska P, Stephenson C, Allen T, Kouskoff V, Lacaud G. The haemangioblast generates haematopoietic cells through a haemogenic endothelium stage. Nature 2009 Feb 12;457 (7231):892-5. [PubMed: 19182774] Time-lapse imaging of differentiating mouse ES cells was used to delineate the developmental progression from the hemangioblast to hemogenic endothelium to hematopoietic cells.

29*. Eilken HM, Nishikawa S, Schroeder T. Continuous single-cell imaging of blood generation from haemogenic endothelium. Nature 2009 Feb 12;457(7231):896-900. [PubMed: 19212410] Using continuous imaging of ES cells at the single-cell level, this study documented that hematopoietic cells arise from the hemangioblast via a hemogenic endothelial intermediate.

30**. Chen MJ, Yokomizo T, Zeigler BM, Dzierzak E, Speck NA. Runx1 is required for the endothelial to haematopoietic cell transition but not thereafter. Nature 2009 Feb 12;457(7231):887-91.

[PubMed: 19129762] Conditional deletion of Runx1 from cells expressing VE-cadherin was used to verify in vivo that HSCs originate from the endothelial cells in hematopoietic organs, including the placenta.

31**. Zovein AC, Hofmann JJ, Lynch M, French WJ, Turlo KA, Yang Y, et al. Fate tracing reveals the endothelial origin of hematopoietic stem cells. Cell Stem Cell 2008 Dec 4;3(6):625-36. [PubMed: 19041779] This study mapped the origin of HSCs in vivo to the endothelial cells of the AGM yolk sac and placenta by labeling derivatives of endothelial cells using a mouse model with temporally restricted induction of Cre from VE-cadherin regulatory elements.

$32 * *$. North TE, Goessling W, Peeters M, Li P, Ceol C, Lord AM, et al. Hematopoietic stem cell development is dependent on blood flow. Cell 2009 May 15;137(4):736-48. [PubMed: 19450519] Using heartbeat deficient sih zebrafish embryos and a zebrafish chemical genetic screen, it was shown that blood flow is required for HSC formation. Nitric oxide signaling emerged as a novel regulator of HSC development in both zebrafish and mouse embryos. 
33**. Adamo L, Naveiras O, Wenzel PL, McKinney-Freeman S, Mack PJ, Gracia-Sancho J, et al. Biomechanical forces promote embryonic haematopoiesis. Nature 2009 Jun 25;459(7250):11315. [PubMed: 19440194] This study presented in vitro and in vivo evidence that shear forces upregulate Runx 1 expression and stimulate hematopoiesis during development in cells derived from mouse ES cells and embryos.

34. Burton GJ, Charnock-Jones DS, Jauniaux E. Regulation of vascular growth and function in the human placenta. Reproduction 2009 Dec;138(6):895-902. [PubMed: 19470597]

35. Chateauvieux S, Ichante JL, Delorme B, Frouin V, Pietu G, Langonne A, et al. Molecular profile of mouse stromal mesenchymal stem cells. Physiol Genomics 2007 Apr 24;29(2):128-38. [PubMed: 17179208]

36. Crisan M, Yap S, Casteilla L, Chen CW, Corselli M, Park TS, et al. A perivascular origin for mesenchymal stem cells in multiple human organs. Cell Stem Cell 2008 Sep 11;3(3):301-13. [PubMed: 18786417]

37. Diez H, Fischer A, Winkler A, Hu CJ, Hatzopoulos AK, Breier G, et al. Hypoxia-mediated activation of Dll4-Notch-Hey2 signaling in endothelial progenitor cells and adoption of arterial cell fate. Exp Cell Res 2007 Jan 1;313(1):1-9. [PubMed: 17045587]

38. Genbacev O, Zhou Y, Ludlow JW, Fisher SJ. Regulation of human placental development by oxygen tension. Science 1997 Sep 12;277(5332):1669-72. [PubMed: 9287221]

\section{Abbreviations}

HSC Hematopoietic stem cell

HS/PC Hematopoietic stem/progenitor cell

AGM Aorta-gonad-mesonephros

MSC Mesenchymal stem cell

VEC Vascular endothelial cadherin 


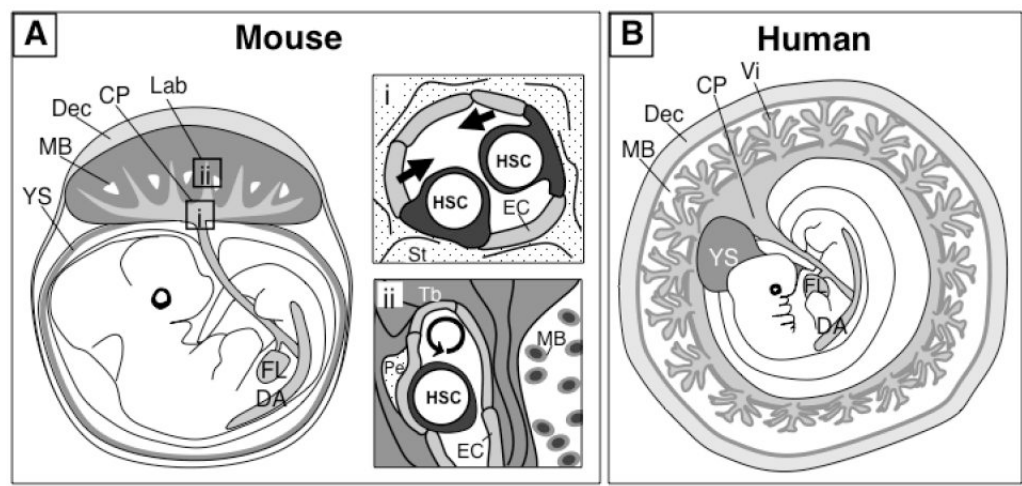

Figure 1. The placenta is a hematopoietic organ that supports generation and proliferation of hematopoietic stem cells (HSC)

A) The mouse placenta consists of two distinct vascular regions that function as putative niches for HSC development. The chorionic plate (CP) harbors large fetal blood vessels that are contiguous with the umbilical cord vessels and connect with the fetus. These blood vessels are potential sites of HSC generation (i). The labyrinth (Lab) region contains an extensive network of small fetal blood vessels that are surrounded by trophoblasts $(\mathrm{Tb})$ lining the maternal blood spaces (MB). The labyrinth is the site of fetal-maternal exchange, and may serve as a niche for HSC expansion (ii). B) The human placenta contains also two vascularized regions, the chorionic plate and the villi (Vi). The villi are physiologically analogous with the labyrinth of the mouse placenta and contain hematopoietic stem/progenitor cells in the vascular and perivascular locations. It is hypothesized that the large vessels of the chorionic plate in the human placenta also have de novo hematopoietic potential. Although the macroscopic organization of the placenta and other extra-embryonic tissues differ between humans and mice, it is likely that similar cellular and molecular mechanisms coordinate placental hematopoiesis in both species. Dorsal aorta (DA), yolk sac (YS), fetal liver (FL), decidua (Dec), endothelial cell (EC), stroma (St), pericyte (Pe). 